\title{
Understanding phase transition in communication networks to enable robust and resilient control ${ }^{\star}$
}

\author{
Soumik Sarkar \\ Kushal Mukherjee \\ Abhishek Srivastav \\ Asok Ray \\ szs200@psu.edu \\ kum162@psu.edu \\ axs964@psu.edu \\ axr2@psu.edu \\ Department of Mechanical Engineering \\ The Pennsylvania State University \\ University Park, PA 16802, USA
}

Keywords: Statistical Mechanics, Thermodynamics, Phase Transition, Communication Networks

\begin{abstract}
This paper presents an application of Statistical Mechanics for analysis of critical phenomena in complex networks. Using the simulation model of a wired communication grid, the nature of phase transition is characterized and the corresponding critical exponent is computed. Network analogs of thermodynamic quantities such as order parameter, temperature, pressure, and composition are defined and the associated network phase diagrams are constructed. The notion of network eutectic point is introduced by showing characteristic similarities between the network phase diagram and the binary phase diagram. A concept of robust and resilient control in communication networks is presented based on network phase diagrams.
\end{abstract}

\section{INTRODUCTION}

A complex network is broadly defined as a collection of interconnected and interacting systems [1], [2] where the individual subsystems (or participating agents) themselves could be complex dynamical systems. Complex networks have been shown to characterize the behavior and topological organization of many natural and engineered systems, such as those found in the disciplines of sociology [3], biology [4], finance [5], and communication networks [6]. A common feature across all such systems is that their global behavior emerges from local dynamics of the participating agents. Specifically, each agent may share its local responsibility as dictated by its neighborhood, which eventually leads to emergence of a global behavior of the whole network. Thus, dynamics of complex networks can be characterized by the relation between micro-motion (i.e., local dynamics) and macro-motion (i.e., global dynamics), which could be characterized by application of the principles of Statistical Mechanics [7], [8]. The underlying similarity of these two disciplines has recently triggered the interest of many researchers to investigate complex networks from the perspectives of Statistical Mechanics; for example, see [1] for a review of recent literature in this field.

\footnotetext{
« This work has been supported in part by the U.S. Army Research Office (ARO) under Grant No. W911NF-07-1-0376, by NASA under Cooperative Agreement No. NNX07AK49A, and by the U.S. Office of Naval Research under Grant No. N00014-08-1-380. Any opinions, findings and conclusions or recommendations expressed in this publication are those of the authors and do not necessarily reflect the views of the sponsoring agencies.
}

A characteristic phenomenon of complex systems, consisting of interacting and interdependent dynamics, is phase transition, where an abrupt (continuous or discontinuous) change in the operating characteristics may take place with a relatively small variation of the system parameter(s). For a communication network, this phenomenon would correspond to dependence of the network's global characteristics (e.g., connectivity and average delay rate) on local parameters (e.g., communication radius and transmission probability) [9]-[11]. This paper presents a Statistical Mechanics approach to study phase transition phenomena in a wired communication network. The qualitative nature of phase transition in the underlying system is characterized and its critical exponent is computed. Phase diagrams are constructed for the network under consideration by defining network analogs of thermodynamic quantities, such as order parameter, temperature, pressure, and composition. The characteristic similarity between the network phase diagram and the binary phase diagram is exploited to define a network eutectic point based on thermodynamic analogy.

The paper is organized as follows. Section II presents a brief background of phase transition and critical phenomena. Using the example of a wired communication network, Section III formulates a Statistical Mechanics approach to study phase transitions. It also investigates the effects of single-parameter and two-parameter variations on initiation of phase transition in a network consisting of single and mixed data types. Section IV discusses potential utilization of phase diagrams for robust and resilient control of communication networks. The paper is concluded with a short discussion and recommendation for future work.

\section{PHASE TRANSITION AND CRITICAL PHENOMENA}

A key task in the analysis of phase transitions is to characterize the system behavior in the vicinity of a critical point. The Mean Field theory is applied to study phase transitions, which involves identification of a global parameter, called the order parameter [7]. It essentially quantifies the presence of order in the underlying system, which is zero (or negligible) in the disordered phase and takes on 
non-zero values in the ordered phase. A phase transition is caused by a continuous or a discontinuous change of the order parameter from zero to a non-zero value when an intensive system parameter (e.g., temperature) is varied across the critical point. For the well-known case of ferromagnetism, the order parameter net-magnetization $M(T)$ has a non-zero value leading to spontaneous magnetization for temperatures below the Curie temperature [7]. The nature of phase transition is often broadly classified into two types $(i)$ first-order phase transition - when the order parameter changes discontinuously with the intensive parameter at the critical point and, (ii) Continuous (also called second or higher order) phase transition - when the order parameter varies continuously with the intensive parameter during the phase transition.

A phase transition is marked by the presence of analytical singularities or discontinuities in the functions describing macroscopic physical parameters of the system. In the vicinity of the critical point marking the phase transition, the functional form of the order parameter is often modeled using a power law with a critical exponent as stated in Eqn. 1 below.

$$
m \sim\left(T_{c}-T\right)^{\beta}
$$

where $m$ is the order parameter; $T$ is the intensive variable (e.g., temperature); $T_{c}$ is the critical value of $T$ corresponding to the phase transition; and $\beta$ is the critical exponent that characterizes the nature of phase transition.

\section{PHASE TRANSITION IN NETWORKS}

Ohira and Sawatari [9] developed a probabilistic routing strategy for a simple wired communication network, which was shown to perform better than a deterministic routing strategy.

\section{A. Example 1: Square-grid wired communication network}

This subsection builds on the concepts presented in Section II to elucidate the phase transition phenomena in wired communication networks based on the Ohira and Sawatari model [9]. Order parameters and network temperature are identified for the network system under consideration; the critical exponent is computed and is compared with the mean-field-theoretic result. A binary network phase diagram is constructed by defining the network composition and different phases are identified. In Section III-B, the model is augmented with the addition of a data type and a network eutectic point is defined.

The network under consideration is a two-dimensional square grid as shown in Fig. 1, where the nodes (routers) are placed at the grid points. For a square grid network with $N \times N$ nodes there are $4(N-1)$ boundary nodes (shown as squares in the Fig. 1) and $N^{2}-4(N-1)$ internal nodes (shown as circles in the Fig. 1). Only boundary nodes are assumed to be the sources and/or the sinks for packet generation and destruction; internal nodes can only transmit the packets. Each node receives packets in an infinite queue from its neighboring nodes and packets

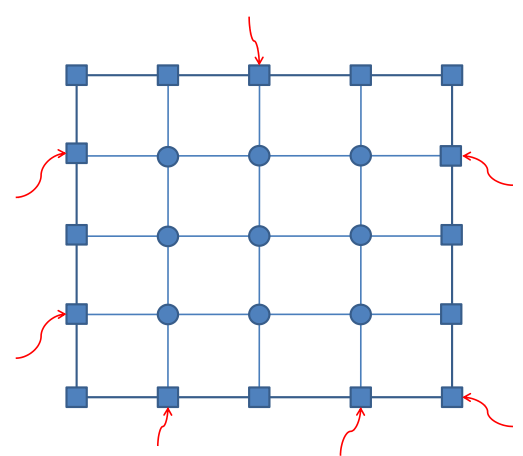

Fig. 1. Network architecture in the simulation model

are terminated after reaching their destinations. In each time unit, packets are created in the boundary nodes with a Poisson arrival rate $\lambda$. Destination nodes are chosen randomly from the boundary nodes, including their source nodes. Each node transmits one packet from the head of its queue to a deterministically chosen neighboring node at each time unit. The node chosen to forward a data packet is selected so that the packet travels via the shortest path to its destination. When there are more than one candidate nodes for the shortest path, the node with a smaller queue length is chosen to prevent early congestion of the network.

Monte Carlo simulation has been performed on a $5 \times 5$ node network. In the simulation model, the wired communication grid is subjected to varying traffic arrival rates $\lambda$ and the network performance is recorded for 300 time units. Average delay $D(t)$ of the network at time instant $t$ is computed as the mean delay of all packets terminating at time $t$. While the average queue length $q(t)$ is computed as the mean queue length of all nodes at time $t$. A typical plot of average delay $D(t)$ and average queue length $q(t)$ with time for arrival rate $\lambda=0.25,0.30,0.35$ and 0.40 is shown in Fig. 2(a) and Fig. 2(b), respectively.

It is seen in Fig. 2 that, after expiry of initial transients, average delay and average queue length come to a steadystate for both $\lambda=0.25$ and $\lambda=0.30$. However, for $\lambda=$ 0.35 and 0.40 , each of $D(t)$ and $q(t)$ does not settle to a steady-state values and shows a consistent rise with a positive rate. The parameters, average delay rate $\left\langle D^{\prime}(t)\right\rangle$ and average queue rate $\left\langle q^{\prime}(t)\right\rangle$, for a given value of $\lambda$ are computed over a span of 300 time units. To eliminate the effects of initial transients in $\left\langle D^{\prime}(t)\right\rangle$ and $\left\langle q^{\prime}(t)\right\rangle$, values of $D(t)$ and $q(t)$ corresponding to first 50 time steps are not included in the computation. Also, for each $\lambda$, delay rate and queue rate of the network are averaged over 10 simulation runs using same system parameters. The plot of $\left\langle D^{\prime}(t)\right\rangle$ and $\left\langle q^{\prime}(t)\right\rangle$ with $\lambda$ is depicted in Fig. 3. It is seen that there is a critical value $\lambda_{c}=0.3$ of the arrival rate such that, for $\lambda<\lambda_{c}$, the average delay rate of data packets is negligible; in contrast, for $\lambda>\lambda_{c}$, average delay rate takes on non-zero values. Similar phenomena are observed for average queue rate of the network. Following Section II, this abrupt change of system behavior across the critical arrival rate $\lambda_{c}$ is identified as a phase transition.

The above discussion and the plots in Fig. 3 evince that the average network delay rate $\left\langle D^{\prime}(t)\right\rangle$ and average queue 


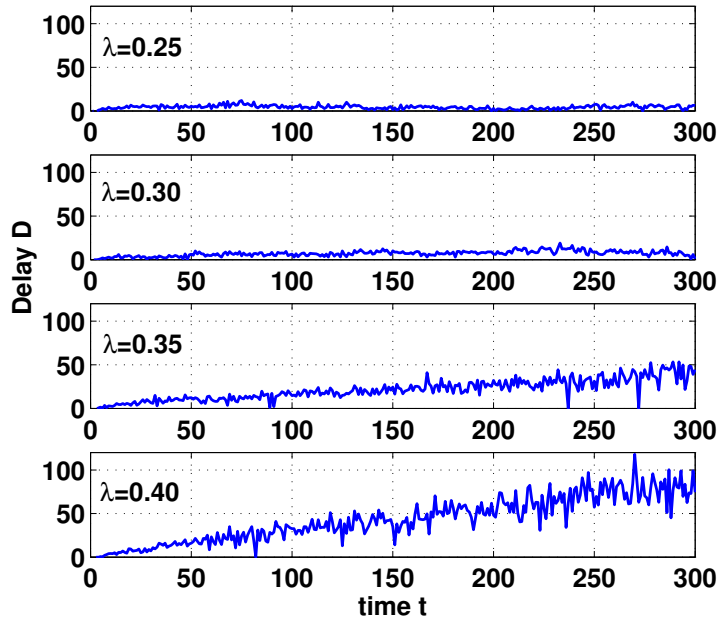

(a)

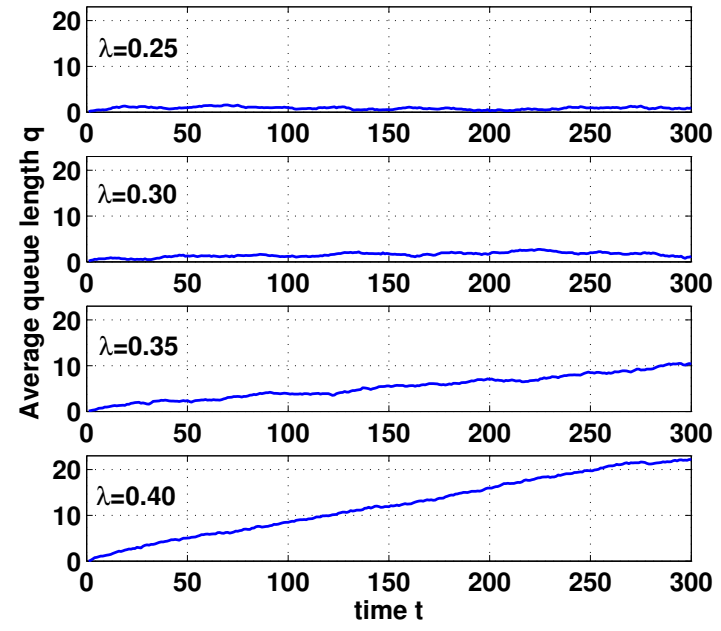

(b)

Fig. 2. Plots of (a) time series of delay $D(t) \quad$ (b) time series of queue length $q(t)$ for arrival rates $\lambda=0.25,0.30,0.35$ and 0.40

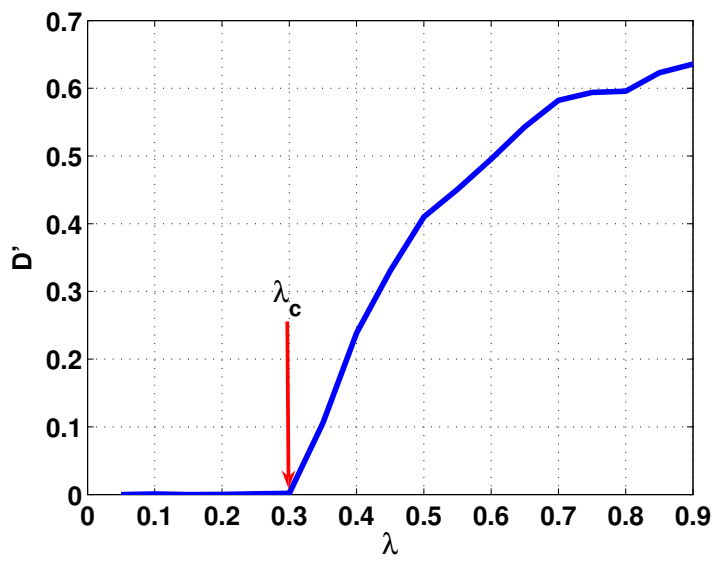

(a)

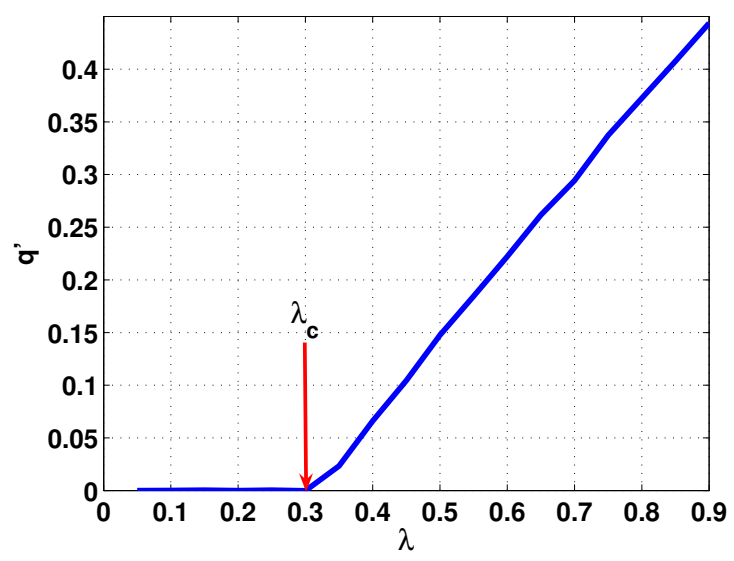

(b)

Fig. 3. Continuous phase transition: (a) average delay rate $\left\langle D^{\prime}(t)\right\rangle$ (b) average queue rate $\left\langle q^{\prime}(t)\right\rangle$ vs. arrival rate $\lambda$; critical arrival rate $\lambda_{c}=0.3$

rate $\left\langle q^{\prime}(t)\right\rangle$ are candidates for network order parameter. With these choices of order parameter and Eqn. (1), the packet arrival rate $\lambda$ is identified as network temperature. The phase transition of the network is of continuous type as defined earlier in Section II. Across the critical point $\lambda_{c}$, the network moves from a stable phase $(\mathbf{S})$ of negligible delay rate and queue rate to an unstable phase (U).

The value of the critical exponent $\beta$ is calculated to be 0.68 and unity for order parameter $\left\langle D^{\prime}(t)\right\rangle$ and $\left\langle q^{\prime}(t)\right\rangle$, respectively, so that $\left\langle D^{\prime}(t)\right\rangle \sim\left|\lambda_{c}-\lambda\right|^{0.68}$ and $\left\langle q^{\prime}(t)\right\rangle \sim$ $\left|\lambda_{c}-\lambda\right|^{1}$, with $\lambda_{c}=0.3$. The same value of $\lambda_{c}$ is expected for both choices of the order parameter. This is justifiable because longer queue lengths imply a higher value of delay for data packets and vice versa.

Previous analyses studied the effects of variation of a single parameter, i.e., packet arrival rate (or network temperature) $\lambda$, on the average delay rate $\left\langle D^{\prime}(t)\right\rangle$. Now, transmission probability $p$ of the nodes, defined as the probability of transmitting a packet by an internal node in a time unit, is varied along with $\lambda$. In the previous case, $p$ was set to unity. When $p$ is decreased from unity, the network is expected to move from the steady delay phase to the unsteady delay phase for lower values of arrival rate $\lambda$. Similar phenomena also take place in thermodynamic (e.g., solid-liquid and gas-liquid) systems, where the critical temperature of solid-liquid (gas-liquid) transition can be altered by a change of super-incumbent pressure; lower pressures lead to lower values of melting (boiling) point. In this regard, the transmission probability $p$ of the nodes is identified as the network pressure. Phase transition in the network is therefore a function of a combination of network temperature $\lambda$ and network pressure $p$.

Simulation runs for different combinations of $\lambda$ and $p$ have been performed. Network stability is determined based on delay rate; and queue rate is negligible for the stable phase and is non-zero for an unstable phase. The 
network phase diagram in Fig. 4 is constructed with $\lambda$ and $p$ as network temperature and pressure, respectively. Stable and unstable regions are labeled as $\mathbf{S}$ and $\mathbf{U}$ respectively in Fig. 4. It is seen that the network phase diagram is characteristically similar to the pressure-temperature phase diagram for ferromagnetic materials for phase transition from ferromagnetism to paramagnetism [7].

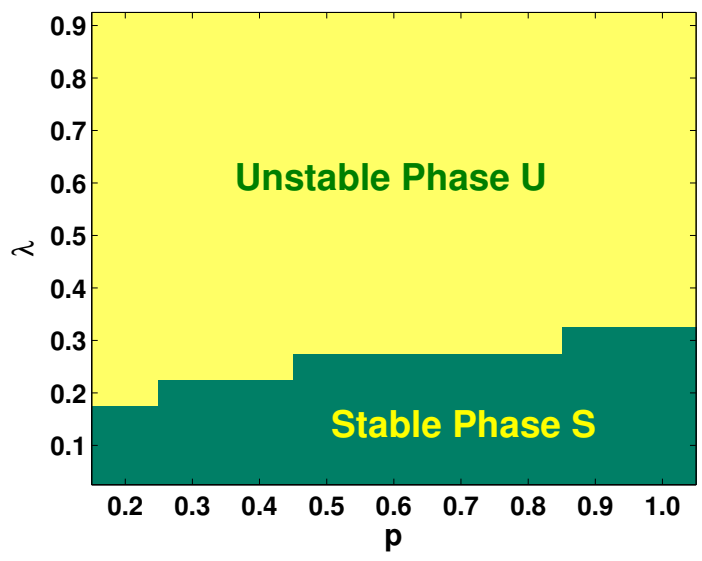

Fig. 4. Network phase diagram

\section{B. Example 2: Square-grid wired communication network with two different data types}

Often, due to limited resources such as channel capacity, data prioritization is used in communication networks to ensure desired performance. The network model studied in the previous section is modified to simulate this situation. Two types of data packets form the network traffic, namely, Type $I$ and Type $I I$. In this example, both data types are created with the same poisson arrival rate $\lambda$. Similar to the model in Section III-A, only nodes at the periphery are assumed to be sources and/or sinks for packet generation and termination; only the nodes internal to the network are allowed to transmit the packets. All nodes have an infinitelength queue for each data type; and data packets received from neighboring nodes are maintained until forwarded to the next node. Packets are terminated after reaching their respective destinations and the routing strategy is kept the same as in Section III-A. Since a node is allowed to transmit only one packet at a time in this model, each node has to choose between two queues for transmitting a packet at each time unit.

Let the priority probability $P_{p}$ of Type $I$ packets be defined as the likelihood with which, a node will choose to transmit data type $I$ at a particular time step. Thus, the probability of transmitting a Type $I I$ packet for a node is $1-P_{p}$ because the transmission probability (network pressure) of each node is maintained at unity. For data traffics of Type I and Type II, each of the three plates in Fig. 5 shows a pair of plots of average queue rate against $\lambda$ for different priority probabilities, (a) $P_{p}=0.3$, (b) 0.5 and (c) 0.9 . Note that, in this model, test cases $P_{p}=0.7$ and 0.1 are equivalent to test cases $P_{p}=0.3$ and 0.9 respectively due to interchangeability of data types and are not shown.

The priority probability $P_{p}$ has a direct influence on the stability of the network with respect to the data types $I$ and $I I$. The critical value $\lambda_{c}$ of arrival rate is seen to be different for different data types. Thus, for different combinations of $\lambda$ and $P_{p}$, it is possible to have one or both data types have a positive delay (queue) rate. It is seen in Fig. 5 that a lower value of $\lambda_{c}$ occurs for lower priority data as expected because a packet of lower priority is less likely to reach its destination and is therefore subjected to higher delays and contributing to longer queues.

As defined earlier, the stable phase $\mathbf{S}$ (unstable phase $\mathbf{U}$ ) for a data type is characterized by zero (non-zero) delay rate and queue rate for that data type. The following four phases of the network are now identified based on this notion.

- Completely Stable phase (S1+S2): Both data types have steady-state delay in this phase, which signifies negligibly small values of $D^{\prime}$ for both channels.

- Unstable data type I and Stable data type II (U1+S2): Data type $I$ has a relatively large $D^{\prime}$ while data type II still maintains a negligibly small $D^{\prime}$.

- Stable data type I and Unstable data type II (S1+U2): Data type $I$ maintains a negligibly small $D^{\prime}$ while Data type $I I$ has a relatively large $D^{\prime}$.

- Completely Unstable phase (U1+U2): Both data types have unsteady delay with large $D^{\prime}$ for each.

A phase diagram is constructed for the network by evaluating the network performance for the two data types at different combinations of $\lambda$ and $P_{p}$. The network phase diagram is shown in Fig. 6 with the four phases of the network defined above. Three locations corresponding to $P_{p}=0.3,0.5$ and 0.9 are marked for which the plot of queue rate $\left\langle q^{\prime}(t)\right\rangle$ with $\lambda$ is given in the plates (a), (b) and (c) of Fig. 5, respectively. In this context, priority probability $P_{p}$ is defined as the network composition as it directly controls the composition of data types in the network traffic.

\section{Material science analogy}

The phase diagram in Fig. 6 is analogous to the binary eutectic phase diagrams in the discipline of Material Science [12] as displayed in Fig. 7. The binary eutectic phase diagram explains the chemical behavior of two immiscible crystals from a completely miscible liquid. For example, let us consider olivine (i.e. isolated tetrahedra) as Crystal A, and pyroxene (i.e. single chain tetrahedra) as Crystal B. Similar to the network phase diagram, temperature is plotted along the ordinate, percentage composition of Crystal A is plotted along the abscissa to construct the binary phase diagram, where pressure is held constant at 1 atmosphere. Note that the network pressure is also held constant in the example in Subsection III-B. The completely stable (i.e., S1+S2) zone in the network phase diagram is analogous to the solid solution (i.e. Crystals 


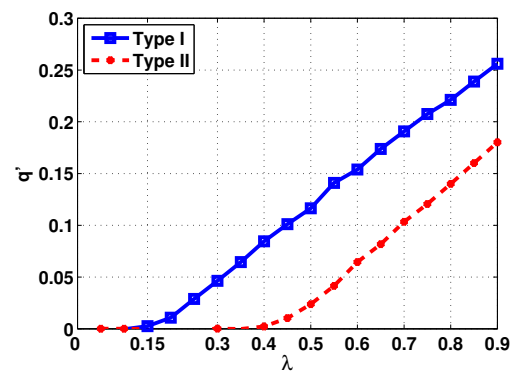

(a)

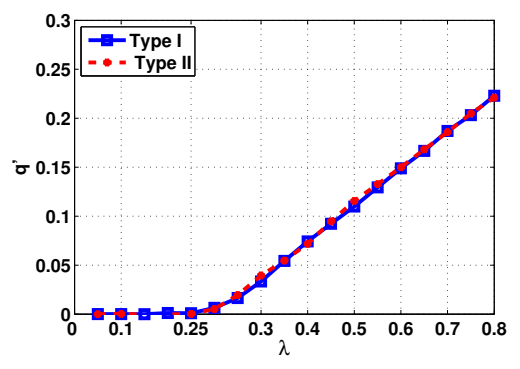

(b)

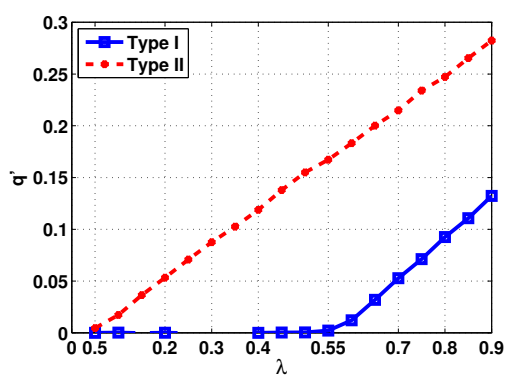

(c)

Fig. 5. Average queue rate $\left\langle q^{\prime}(t)\right\rangle$ vs. arrival rate $\lambda$ with mixed traffic:

(a) $P_{p}=0.3$

(b) $P_{p}=0.5$

(c) $P_{p}=0.9$

$\mathrm{A}+\mathrm{B})$ zone of the binary phase diagram in Fig. 7. While the completely Unstable (U1+U2) zone in Fig. 6 is analogous to the "All Melt" zone in Fig. 7. Similarly, zones (U1+S2) and $(\mathbf{S 1}+\mathbf{U} 2)$ are analogous to the "Melt+A" and "Melt+B" zones respectively, where "Melt $+\mathrm{A}$ " signifies a phase with molten mixture and crystals of A in it; and similar analogy hold for "Melt+B". Equivalent solidus and liquidus lines can also be drawn for network phase diagram, which will denote the boundaries between different phases.

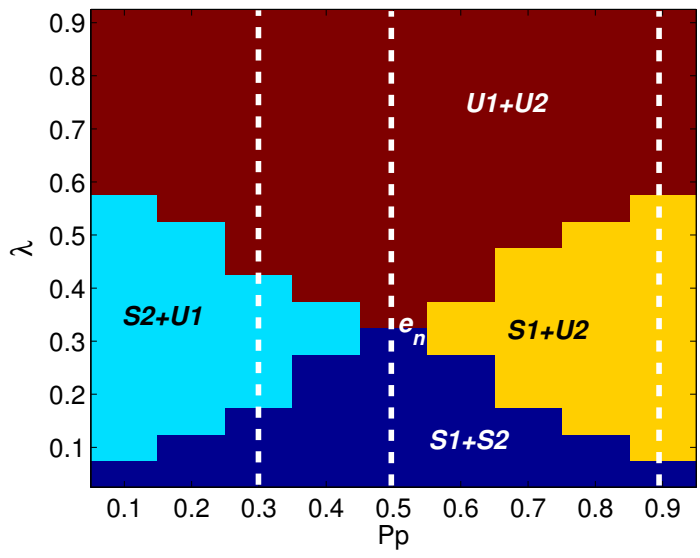

Fig. 6. Network phase diagram for mixed traffic

\section{NETWORK ROBUSTNESS AND RESILIENCE}

Robustness of a dynamical system signifies its insensitivity to exogenous disturbances, internal faults, and uncertainties. In this context, robust decision \& control laws are synthesized with the objectives of achieving optimal trade-offs between stability and performance under disturbances and (both structured and unstructured) uncertainties within specified bounds [13]. For the binary phase diagram described above, the melting point of the eutectic mixture is as low as possible. The corresponding melting temperature is called the eutectic temperature, and the composition and temperature at which melting takes place is called the eutectic point as 'e' in Fig. 7. Therefore, when reducing the temperature across the eutectic point, both constituents crystallize simultaneously from the molten liquid solution. Similarly, a network eutectic point $e_{n}$ is

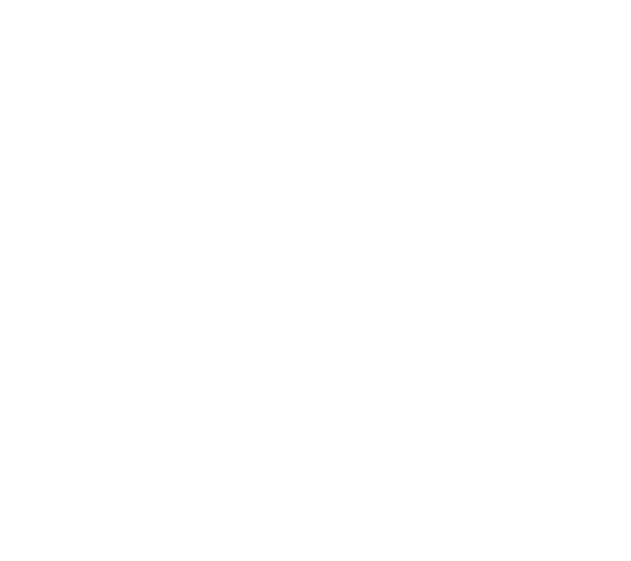

Fig. 7. Binary phase diagram for mixed crystals

defined as the point at which both data types move to their unstable phases when the network temperature (i.e., packet arrival rate $\lambda$ ) is increased keeping the network composition constant. Thus, the corresponding $\lambda$ would be highest amount of traffic that can be accommodated with both data types stable to achieve the highest performance. However, due to some disturbances or fluctuations in the network traffic if this point is crossed, then both the traffic types will become unstable. Therefore, qualitatively, this phase diagram dictates that, for robustness of the network the operating point, should not be in the vicinity of the network eutectic point.

While network robustness is relevant in the infinite-time horizon, network resilience needs to be considered in a a finite-time horizon. The role of a resilient controller is to accommodate unanticipated adverse conditions and large faults as well as emergency situations by altering its operational envelope in real time [14]. Upon reaching normalcy, the network system must return to the original state of operation or a gracefully degraded state in the event of a permanent damage.

As an example, a real-life situation is presented in this paper to elucidate the concept of network resilience. Let us consider a sensor network deployed to collect video and chemical data from a monitored environment. Depending on the situation at hand, the video or the chemical data 


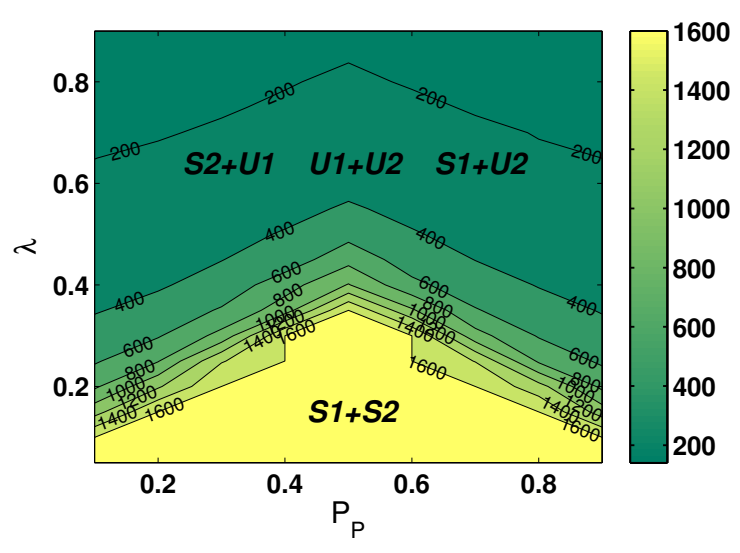

Fig. 8. Network resilience - time units that the network is allowed to stay in the unstable zone

would be of higher priority. Chemical sensor data that are less bulky compared to video sensor data would be expected to be assigned higher priority. However, this policy may change in an emergency situation when a resilient network controller is required to opportunistically assign the priorities to individual data types. In an attempt to do so, the network may possibly visit the unstable phase of low priority data for a short duration to allow the timecritical data to reach its destination fast enough. Upon accomplishing this task, the network should autonomously return to the stable phase for both data types. A plot of allowed time units that the network can spend in a phase before becoming unstable is shown in Fig. 8. This information is computed as the time before the average queue lengths become larger than a predefined bound, which is chosen to be 40 in this example. Since the network average queue rate is approximately zero in the stable phase (i.e., $\mathbf{S 1 + S 2 )}$, the network can operate indefinitely without filling up the queues. However, in an unstable phase (i.e., $\mathbf{S 1 + U 2 ,}$ $\mathbf{U 1 + S 2}$ or $\mathbf{U 1 +} \mathbf{U} 2)$, there is a specified number of time units, shown as contours lines in Fig. 8, before which priorities of individual data types should be altered to maintain the queue lengths within the specified bound. Thus, depending on the current needs of data transmission, the network prioritizes the data based on the network phase diagram and choose the network composition for resilient control.

\section{CONCLUSIONS AND FUTURE WORK}

Phase transition in communication networks are considered and the associated phase diagrams of network operation are constructed. In this context, network analogues of order parameter, temperature, pressure, and composition are defined along with the concept of the network eutectic point. To elucidate this concept, the model of a wired communication network is considered with single data type and a mixture of two different data types for Monte Carlo simulation. A binary phase diagram is constructed for the network with a mixture of two different data types.
Significance of the network phase diagram is discussed to achieve robustness and resilience for network control.

Further research is needed before the Statistical Mechanics concept, presented in this paper, can be considered for analysis and synthesis of large-scale networks. While there are many research areas to be investigated, the following topics are being currently pursued.

- Concepts of Statistical Mechanics such as the renormalization group [15] [16] for modeling phase transition in multi-time-scale network operations.

- Investigation of more complex topologies as needed for large-scale communication networks and sensor networks.

- Development of Statistical Mechanics inspired routing algorithm for robust and resilient control of large scale sensor networks.

\section{REFERENCES}

[1] R. Albert and A.-L. Barabasi, "Statistical mechanics of complex networks," Review of Modern Physics, vol. 74, pp. 47-97, January 2002.

[2] R. Pastor-Satorras, J.-M. Rubi, and A. Diaz-Guilera, Statistical Mechanics of Complex Networks. New York, NY, USA: Springer, 2003.

[3] S. Durlauf, "How can statistical mechanics contribute to social science?" Proc. Natl. Acad. Sci. USA, vol. 96, p. 1058210584, September 1999.

[4] M. Millonas, "Swarms, phase transitions, and collective intelligence (paper 1); and a nonequilibrium statistical field theory of swarms and other spatially extended complex systems (paper 2)," Santa Fe Institute, Working Papers 93-06-039, Jun. 1993. [Online]. Available: http://ideas.repec.org/p/wop/safiwp/93-06-039.html

[5] M. Schulz, Statistical Physics and Economics: Concepts, Tools and Applications Springer Tracts in Modern Physics, Vol. 184. Berlin, Germany: Springer, 2003.

[6] B. Krishnamachari, S. Wicker, and R. Bejar, "Phase transition phenomena in wireless ad-hoc networks," in Global Telecommunications Conference, IEEE, 2001.

[7] R. Pathria, Statistical Mechanics, 2nd ed. Oxford, UK Butterworth-Heinemann, 1996.

[8] K. Huang, Statistical Mechanics, 2nd ed. New York, NY, USA John Wiley \& Sons, 1987.

[9] T. Ohira and R. Sawatari, "Phase transition in computer network traffic model," Physical Review E, vol. 58, pp. 193-195, 1998.

[10] A. Lawniczak and X. Tang, "Network traffic behaviour near phase transition point," The European Physical Journal B - Condensed Matter and Complex Systems, vol. 50, no. 1-2, pp. 231-236.

[11] A. Lawniczak, P. Lio, S. Xie, and J. Xu, "Wavelet spectral analysis of packet traffic near phase transition point from free flow to congestion in data network model," Canadian Conference on Electrical and Computer Engineering, CCECE 2007., pp. 364-367, April 2007.

[12] L. Fichter, "Binary eutectic phase diagram," Department of Geology and Environmental Science, James Madison University, Harrisonburg, Virginia 22807, Tech. Rep., 2000. [Online]. Available: http://csmres.jmu.edu/geollab/Fichter/IgnRx/BinryEu.html

[13] K. Zhou, Robust and Optimal Control. Upper Saddle River, NJ, USA: Prentice-Hall, 1996.

[14] T.-H. Guo and J. Litt, "Resilient propulsion control research for the NASA Integrated Resilient Aircraft Control (IRAC) Project," in Proceedings of AIAA 2007-2802, Infotech Aerospace Conference, 2007

[15] K. G. Wilson, "Renormalization group and critical phenomena. i. renormalization group and the kadanoff scaling picture," Phys. Rev. $B-4$, p. $3174,1971$.

[16] — - "Renormalization group and critical phenomena. ii. phasespace cell analysis of critical behavior," Phys. Rev. B-4, p. 3184, 1971 